\title{
Isolated theropod teeth from the Middle Jurassic of Niger and the early dental evolution of Spinosauridae
}

\author{
Alejandro Serrano-Martínez, Daniel Vidal, Lara Scisio, Francisco Ortega, and Fabien Knoll \\ Acta Palaeontologica Polonica 61 (2), 2016: $403-415$ doi:http://dx.doi.org/10.4202/app.00101.2014
}

Four isolated theropod teeth from the ?Bathonian "Argiles de l'Irhazer" in Niger are described. The teeth were found in association with the holotype of the basal sauropod Spinophorosaurus nigerensis. These specimens have been assigned to two different taxa by independent analyses, such as direct comparison with teeth previously described in the literature, discriminant and morphometric analyses from metric characters, and cladistic and cluster analyses from discrete characters. The results suggest that three teeth share affinities with those of Megalosauridae and Allosauridae, belonging most likely to the former. The fourth tooth might be from a member of the stem group Spinosauridae. If so, this would be the oldest representative of this clade. This tooth shows a combination of characters that are unusual in typical spinosaurid teeth (crown moderately compressed labiolingually and curved distally with minute denticles on the carina and a deeply veined enamel surface texture without apicobasal ridges). This could shed light on the morphological transition from the plesiomorphic ziphodont dental pattern to that of Spinosauridae. This tooth would also allow a better understanding of the origin of the spinosaurids, supporting a Gondwanan origin for the group.

Key words: Theropoda, Megalosauridae, Spinosauridae, dental morphology, multivariate analyses, Jurassic, Africa.

Alejandro Serrano-Martínez [a.serrano@ccia.uned.es], Grupo de Biología Evolutiva, Facultad de Ciencias, UNED, Madrid, Spain; Departamento de Biología,

Facultad de Ciencias, Universidad Autónoma de Madrid, Madrid, Spain;

Departamento de Paleobiología, Museo Nacional de Ciencias Naturales-CSIC, Madrid, Spain. Daniel Vidal [rickraptor@me.com], Departamento de Biología,

Facultad de Ciencias, Universidad Autónoma de Madrid, Madrid, Spain. Lara

Scisio [scslar001@myuct.ac.za] Department of Geological Sciences, University of Cape Town, South Africa. Francisco Ortega [fortega@ccia.uned.es] Grupo de Biología Evolutiva, Facultad de Ciencias, UNED, Madrid, Spain. Fabien Knoll [knoll@mncn.csic.es , fabien.knoll@manchester.ac.uk, fabien.knoll@bristol.ac.uk] Departamento de Paleobiología, Museo Nacional de Ciencias Naturales-CSIC, Madrid, Spain; School of Earth, Atmospheric and Environmental Sciences, University of Manchester, Oxford Road, Manchester, M13 9PL, United Kingdom; School of Earth Sciences, University of Bristol, 24 Tyndall Avenue, Bristol, BS8 1TQ, United Kingdom. 
This is an open-access article distributed under the terms of the Creative Commons Attribution License (for details please see creativecommons.org), which permits unrestricted use, distribution, and reproduction in any medium, provided the original author and source are credited.

FaF Full text $(791.5 \mathrm{kB})$ ।

Far 5 Supplementary file $(142.7 \mathrm{kB})$ 